\section{Rapport annuel du CSPV 1999}

\author{
M. Kuhn, K. Hartmann
}

En 1999, le CSPV a enregistré pour la première fois plus de mille notifications d'effets indésirables suspectés (EI) reçues directement de membres des professions médicales. Ces 1058 cas représentent une augmentation de 13,3\% par rapport à 1998, une hausse sensible que nous considérons comme l'illustration de la confiance dont notre Centre jouit auprès des professions de la santé. Le premier commentaire que l'auteur de la notification reçoit dans les trois jours ouvrables est particulièrement apprécié. Cette rapide évaluation personnalisée de chaque cas constitue pour le médecin ou le pharmacien en question une sorte de formation continue "à chaud", elle contribue à l'assurance de qualité et à l'amélioration de la sécurité du médicament.

\section{Organisation du travail}

La communication par Internet exerce une influence toujours plus grande sur le travail du CSPV. Sa page d'accueil (http://www.hin.ch/sanz) a été développée. La formule de notification qui s'y trouve facilite sensiblement la communication d'un rapport. Sur demande, le suivi du cas et la correspondance y relative peuvent aussi se transmettre par cette voie. Cette page signale également les dernières publications du Centre, elle permet de les consulter et de les imprimer.

$\mathrm{Au}$ cours de l'exercice, toutes les connexions du CSPV ont été adaptées et modernisées afin de rendre encore plus performantes les liaisons électroniques, notamment avec les autorités.

\section{Hémovigilance}

Le nombre d'effets secondaires suspectés dans ce domaine s'est élevé à 26 durant l'exercice, contre 18 en 1998.

Correspondance:

Max Kuhn

CSPV

Neubruchstrasse 37

$\mathrm{CH}-7000$ Coire
En sa qualité de membre associé, le docteur Brigit Brand a participé à plusieurs séances du «European Haemovigilance Network" pour faire part des expériences suisses et recueillir celles des centres étrangers.

\section{Collaboration avec les autorités}

La nouvelle convention avec l'OFSP est entrée en vigueur le 1.1.99, elle règle l'échange anonymisé des données sur les effets secondaires suspectés à la suite de vaccinations. Les enquêtes minutieuses du CSPV sur ces cas constituent pour l'OFSP une aide décisionnelle en vue d'éventuelles mesures sanitaires. Lors d'un symposium en mars à Zoug sur les vaccinations, le CSPV a pu présenter à un nombreux auditoire son expérience de plusieurs années.

Le CSPV collabore depuis 1991 étroitement avec l'OICM, auquel il transmet sous forme anonymisée les EI suspectés jugés valables, soit 858 rapports durant l'exercice (700 en 1998). Plusieurs entretiens concernant la sécurité du médicament ont eu lieu avec les représentants de l'autorité.

La nouvelle Loi sur les produits thérapeutiques figure à l'ordre du jour des délibérations parlementaires en l'an 2000. A la place de l'Office intercantonal, ce sera un Institut suisse qui sera compétent pour toutes les questions des médicaments et de leur sécurité. Tous les membres des professions médicales seront tenus de notifier les EI graves, ce qui implique une réorganisation de la pharmacovigilance en Suisse.

Au printemps 1999, le concept présenté était tellement flou sur le rôle du CSPV que les organisations donatrices de ce dernier ont fait savoir que leur concours financier ne subsisterait que si les autorités manifestaient la volonté expresse de confier au CSPV un mandat concernant le système de notifications spontanées. Le projet de Loi sur les produits thérapeutiques prévoit en effet une délégation d'activités à des fondations privées en cas d'avantage économique.

Le 12 mai 1999, les conseil de fondation du CSPV a publié dans les organes officiels des médecins et des pharmaciens qu'il serait contraint d'arrêter l'activité du Centre si les autorités n'indiquaient pas clairement leurs intentions à ce sujet. En l'espace de trois semaines, quelque 900 médecins et plus de 100 pharmaciens ont demandé le maintien du CSPV dont les prestations sont appréciées. Des entretiens approfondis et constructifs ont eu lieu entre-temps avec les représentants de l’OICM. Le CSPV est convaincu qu'il continuera à jouer un rôle important dans le système suisse de notifications spontanées et que ses services resteront comme auparavant à disposition des membres des professions médicales. 


\section{Figure 1}

Origine des notifications.

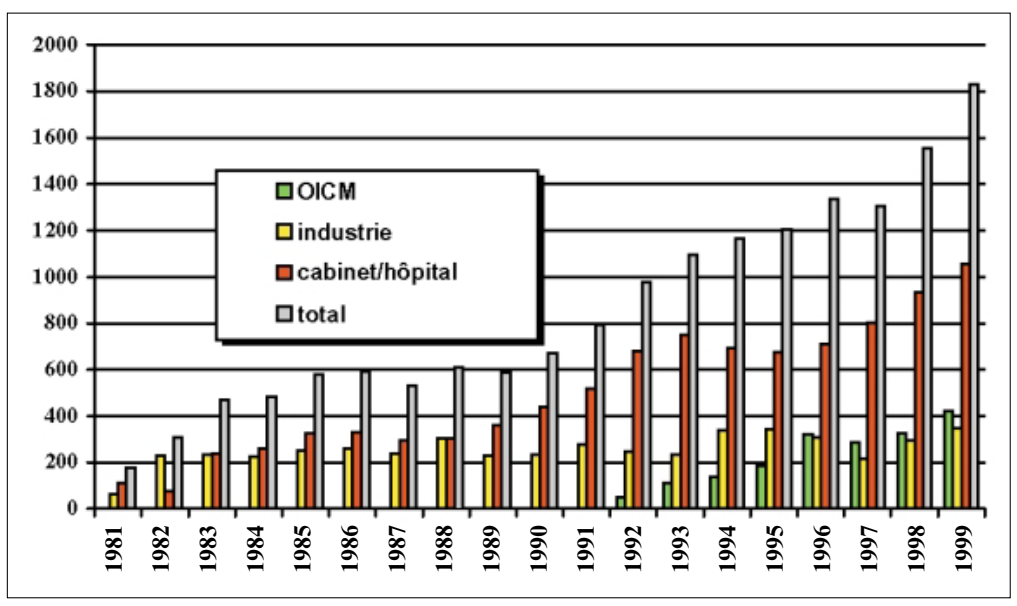

\section{Statistiques}

Durant l'exercice, 1828 nouveaux cas ont été enregistrés dans la banque de données du CSPV. Le nombre de ceux signalés directement par des médecins et pharmaciens est en nette augmentation, soit 1058 contre 934 en 1998. Les notifications provenant de l'industrie ont augmenté de 296 en 1998 à 346, elles concernent des cas signalés à l'entreprise par les médecins et les pharmaciens. La centrale d'effets indésirables de l'OICM a communiqué 424 cas au CSPV (325 en 1998). Le total s'élève donc à 1828 cas noti- fiés. La figure 1 montre l'évolution de ces chiffres depuis la fondation du CSPV en 1981.

De tous les rapports que le CSPV a reçu des professions médicales en 1999, 62\% proviennent de médecins privés, 20,3\% d'hôpitaux cantonaux et régionaux, 5\% de cliniques universitaires, 5,9\% de pharmaciens et 6,8\% de médecins dentistes, médecins à la retraite, médecins militaires et d'assurances.

Les réactions d'hypersensibilité, c'est-à-dire les symptômes affectant le corps en général, dominent. Suivent les EI concernant la peau, le système nerveux, le tractus digestif, le psychisme et le système cardiovasculaire. Le nombre total d'effets indésirables est plus élevé que celui des rapports, car plusieurs systèmes peuvent participer au tableau clinique d'un seul cas. La répartition s'avère assez constante d'une année à l'autre (figure 2).

La figure 3 indique les groupes de principes actifs les plus fréquemment mis en cause. Là aussi, le nombre de médicaments est supérieur à celui des rapports, car plusieurs principes actifs peuvent être à l'origine des EI d'un seul et même cas. Les antiinflammatoires non-stéroïdiens (AINS) figurent cette année pour la première fois en tête, ce qui est dû au grand nombre de notifications concernant les nouveaux inhibiteurs de la COX-2. Cela ne signifie pas pour autant que ces substances provoquent plus fréquemment des effets secondaires, mais illustre une caractéristique majeure du système de notifications spontanées, à savoir que les nouveaux médicaments sont surveillés plus étroitement par les professions de santé et font dès lors l'objet de notifications plus fréquentes et plus rapides. Or, si ces principes actifs at-

Figure 2

Répartition des EI notifiés en fonction des systèmes d'organes.

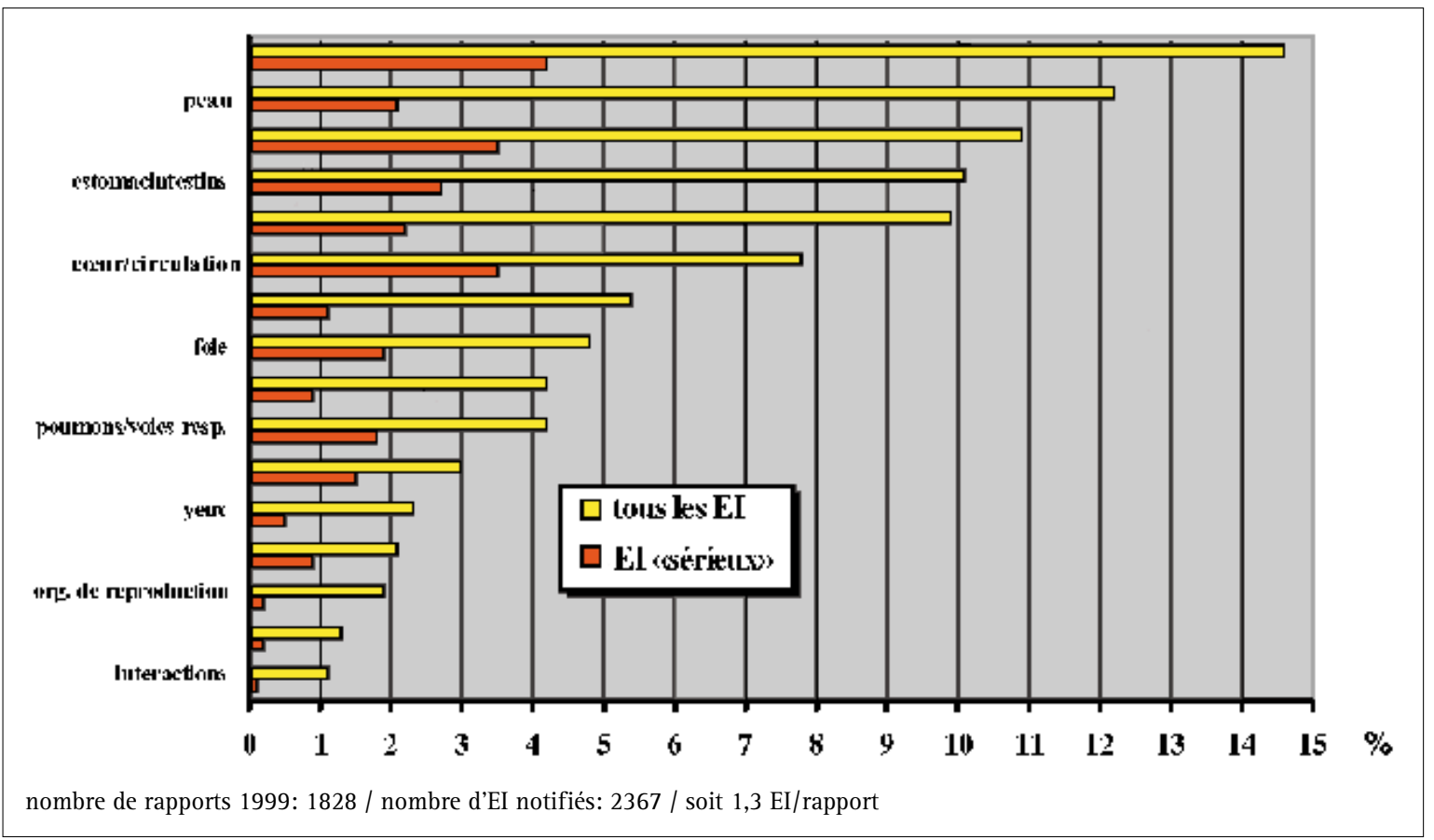


Figure 3

Répartition des EI notifiés selon groupe de principes actifs.

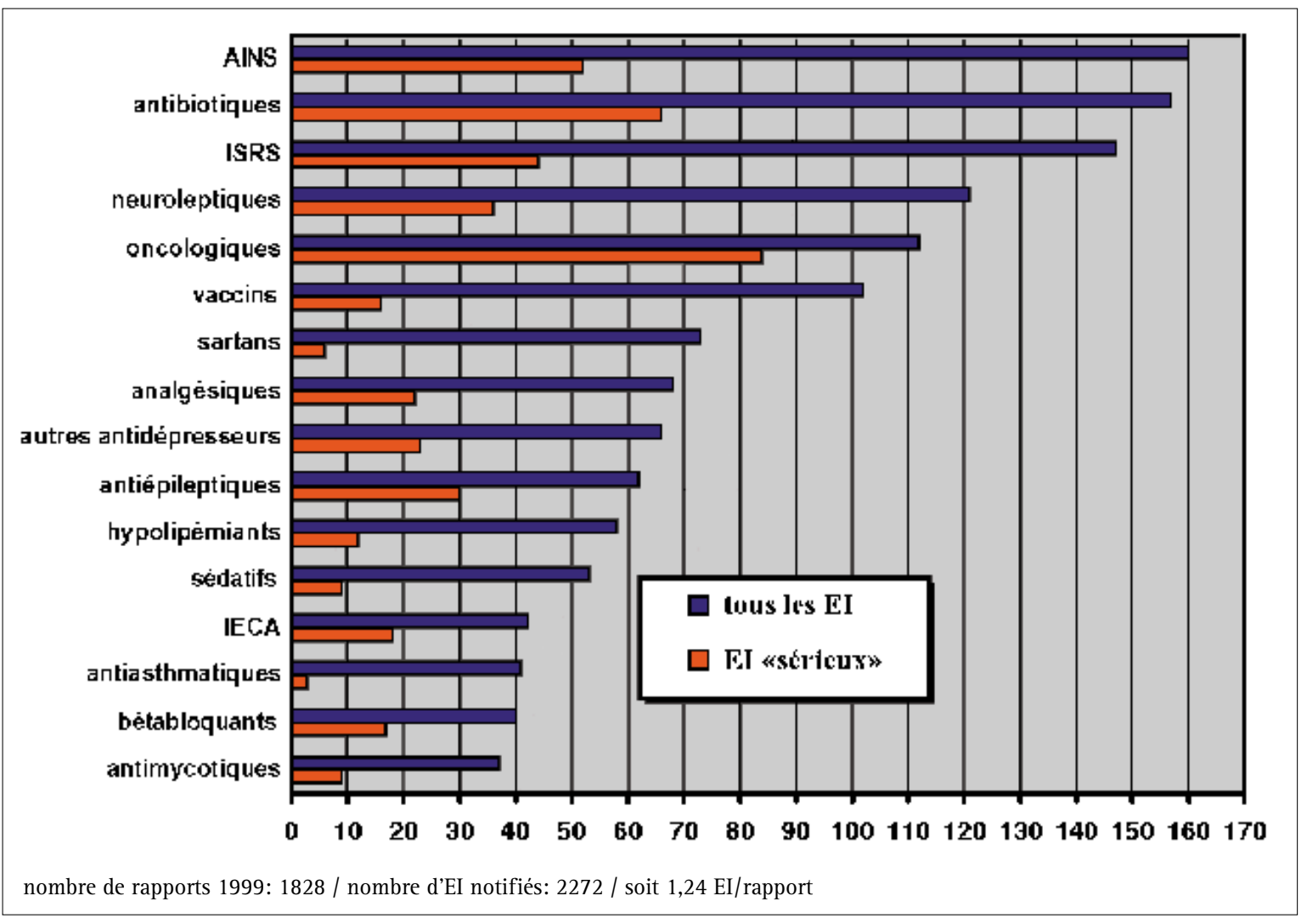

teignent rapidement une part notable du marché, le nombre de rapports s'élève en conséquence.

Suivent dans l'ordre les antibiotiques, les inhibiteurs sélectifs du recaptage de la sérotonine, les neuroleptiques et les principes actifs utilisés en oncologie. En ce qui concerne les vaccins, il s'agit surtout de réactions locales.

Nous avons enregistré cette année 30,5\% d'EI considérés comme sévères, "sérieux" selon les critères internationaux. Dans 60 cas (56 en 1998), la personne ayant notifié estime qu'un médicament pourrait jouer un rôle dans les causes de décès. Ces cas sont d'une grande valeur pour l'OICM, autorité d'enregistrement, et pour la sécurité du médicament en général. Dans 35 cas (1,9\% de toutes les notifications) une relation causale est jugée au moins "possible».

La figure 4 indique l'origine des rapports sur des EI graves ou d'issue fatale.

La plupart des rapports que reçoit le CSPV en 1999 provient une fois de plus d'une liaison directe avec les membres de professions médicales. Or, dans le système de notifications spontanées, ces cas graves revêtent une grande importance et leur fonction de signal d'alerte est primordiale.

Les patients concernés sont de sexe masculin pour $44,5 \%$ et de sexe féminin pour 55,5\%.

Les notifications proviennent de Suisse allemande pour $80 \%$, de Romandie pour $17 \%$ et du Tessin pour $3 \%$ des cas.

\section{Publications en 1999}

\section{Organisation et activité du CSPV}

Kuhn M, Hartmann K. CSPV - Rapport annuel 1998. Bull méd suisses 1999;80:1309-11.

Publications concernant des effets indésirables Hartmann K, Koller Doser A, Kuhn M. Reflets du CSPV n ${ }^{\circ} 37$ : Valeur et utilité pour la sécurité du médicament des notifications spontanées d'effets indésirables (EI). Bull méd suisses 1999;80:917-9; J suisse pharm 1999;137:80-1.

Courtin C, Hartmann K, Reinhart WH, Kuhn M. Reflets du CSPV $\mathrm{n}^{\circ}$ 38: Herbalife ${ }^{\circ}$ - réactions d'hypersensibilité. Problèmes que pose l'achat on-line. Bull méd suisses 1999;80:983-4; J suisse pharm 1999;137:212-4.

Hartmann G, Hartmann K, Kuhn M. Reflets du CSPV n 39: Notifications d'effets indésirables de statines depuis leur introduction en 1989 jusqu'en septembre 1998. Bull méd suisses 1999;80:2067-9; J suisse pharm 1999;137:400-2.

Bon S, Hartmann K, Kuhn M. Reflets du CSPV n 40: Le millepertuis, un inducteur enzymatique? Bull méd suisses 1999;80: 2195-6; J suisse pharm 1999;137:537-8.

Schuster C, Reinhart WH, Hartmann K, Kuhn M. Angioödem unter ACE-Hemmern und Angiotensin-II-Rezeptor-Antagonisten: Analyse von 98 Fällen. J suisse méd 1999;129:362-9.

Gaudenz R, Hartmann K, Reinhart WH, Kuhn M. Ausgedehnte eosinophile Lungeninfiltrate unter antidepressiver Behandlung mit Maprotilin. Revue méd suisse (Praxis) 1999;88:1047-51. 


\section{Figure 4}

Nombre de notifications d'EI sévères ("sérieux») adressées au CSPV, à l'OICM et à l'industrie.

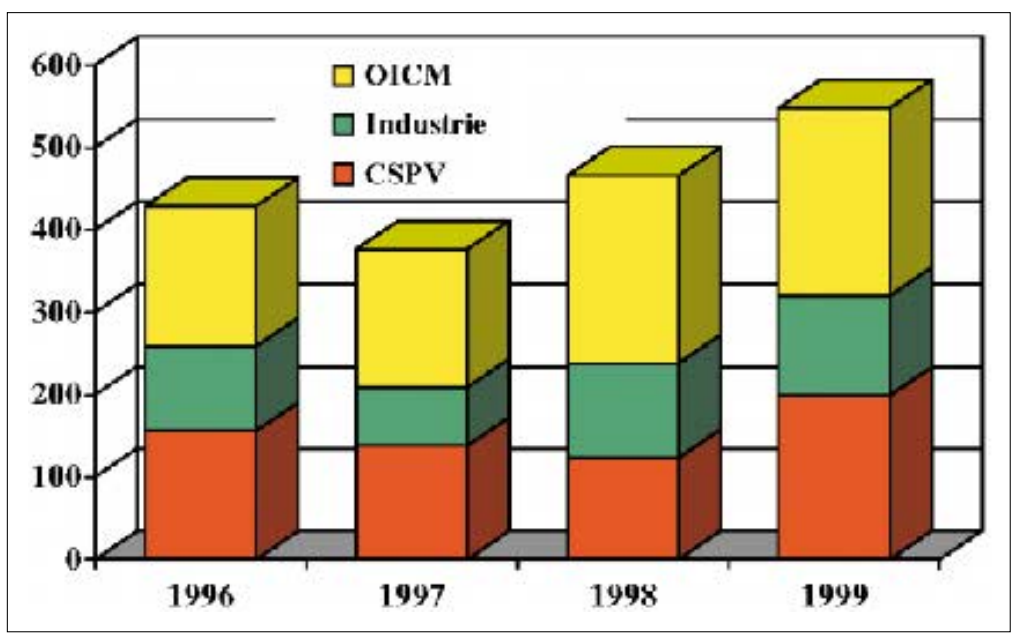

Walter R, Hartmann K, Fleisch F, Reinhart WH, Kuhn M. Reactivation of herpesvirus infections after vaccinations? Lancet 1999;353:810.

Bielecki JW, Schraner C, Briner V, Kuhn M. Rhabdomyolyse und cholestatische Hepatitis unter der Behandlung mit Simvastatin und Chlorzoxazon. J suisse méd 1999;129:514-8.

Meyer MI, Kuhn M, Bühler H, Bertschinger P. Ticlopidin-induzierte Cholestase. J suisse méd 1999;129:1405-9.

Hartmann K, Koller Doser A, Kuhn M. Postmarketing Safety Information: How useful are spontaneous reports? Pharmacoepidemiology and Drug Safety 1999;8:S65-S71.

\section{Contributions à des congrès}

Kuhn M. Impfkomplikationen: Was nun? Journée de formation continue. Problèmes de vaccination en pratique quotidienne. Casino Zoug; Zoug; 4.3.1999.

Schuster C, Reinhart WH, Hartmann K, Kuhn M. Angioödem unter ACE-Hemmern und Angiotensin-II-Rezeptor-Antagonisten: Analyse von 98 Fällen. Posterpräsentation an der 67. Jahresversammlung der Schweizerischen Gesellschaft für Innere Medizin, Basel, 15.-17. April 1999. J suisse méd 1999; 129(Suppl 105/I):32 S.

Walter R, Hartmann K, Fleisch F, Reinhart WH, Kuhn M. Reaktivierte Herpes-Infektionen nach Impfungen. Posterpräsentation an der 67. Jahresversammlung der Schweizerischen Gesellschaft für Infektiologie, Basel, 15.-17. April 1999. J suisse méd 1999;129(Suppl 105/II):15 S.

Courtin C, Hartmann K, Reinhart WH, Kuhn M. Herbalife ${ }^{\circledR}$ Überempfindlichkeitsreaktionen oder Problematik der OnlineMedikamentenbeschaffung. Posterpräsentation an der 67. Jahresversammlung der Schweizerischen Gesellschaft für Klinische Pharmakologie, Basel, 15.-17. April 1999. J suisse méd 1999;129(Suppl 105/II):33 S.

Hartmann K, Pool V, Walter R, Gargiullo P, Kuhn M. Reactivation of Herpes Infections after Vaccinations? Alerts from Spontaneous Reports and Hypothesis Testing. Posterpresentation at the $15^{\text {th }}$ International Conference on Pharmacoepidemiology, Boston, August 26-29 1999. Pharmacoepidemiology and Drug Safety 1999; 8(Suppl 2):S124.

Brand B, Hartmann K, Kuhn M. Haemovigilance experience since 1996 in Switzerland. 32 Congrès annuel de la Société allemande de médecine de transfusion. 5-8 octobre 1999, Münster (Westphalie)/D. 